\title{
Rising Trends of Obesity in Malaysia; Role of Inflammation and Inflammatory Markers in Obesity Related Insulin Resistance: A Nuclear Factor Kappa B (Nfkb) Perspective
}

\author{
Ilyas $\mathrm{MN}^{1}$, Atif $\mathrm{AB}^{1 *}$, Al-Hatamleh $\mathrm{MAI}^{2}$, Al-Shajrawi $\mathrm{OM}^{1}$, Ariff $\mathrm{TM}^{2}$ and Nordin Simbak \\ ${ }^{1}$ Faculty of Medicine, University Sultan Zainal Abidin, Malaysia \\ ${ }^{2}$ CODE- Universiti Sultan Zainal Abidin, Malaysia
}

Submission: November 07, 2017; Published: December 15, 2017

*Corresponding author: Atif AB, Faculty of Medicine, Universiti Sultan Zainal Abidin, Malaysia, Email: atifamin@unisza.edu.my

\section{Obesity and Insullin Resistance}

Obesity is a condition with excessive and abnormal fat accumulation in the body. Overweight and obesity is now a widespread problem and presents as a risk factor for chronic diseases, like diabetes, heart diseases and cancers [1]. Mainly, a population measure of obesity is the body mass index (BMI), a person's weight (kilogram) divided by the square of his or her height (meters) [2]. Normally, individual with BMI equal to or more than 30 is considered obese [2]. Obesity is a worldwide problem and major risk factor for multiple disease complications like insulin resistance.

Insulin resistance is defined clinically as the inability of insulin to increase glucose uptake and utilization in an individual. Insulin resistance is the consequence of obesity and other causes which impair signalling pathways [3]. It is believed that obesityrelated chronic inflammation at the cellular level is responsible for the decrease in insulin sensitivity [4]. The decrease in insulin sensitivity makes obesity a major risk for the development of insulin resistance related disorders like type 2 diabetes and metabolic syndrome [5]. The main source for inflammation induced obesity and insulin resistance were reported, are tissue hypoxia, excess nutrients and adipocyte dysfunction [6]. The researcher identified that hypoxia via hypoxia-inducible factor $1 \alpha$ (HIF- $1 \alpha)$ and other signalling mechanism might enhance the free fatty acid release and inhibit glucose uptake in adipocytes [6]. Hypoxia also triggers the nucleus translocation of nuclear factor kappa $\beta$ (NF- $\kappa B$ ), which initiate the regulation of genes that encode inflammatory cytokines. These cytokines i.e. TNF- $\alpha$, IL-6, and IL-1 $\beta$ are involved in adipose tissue inflammation [7].

Chronic inflammation is also caused by over eating that leads to excessive lipid accumulation in adipocytes [4]. Obesity due to overeating might activate inflammatory signalling pathways by increasing expression of inflammatory cytokines. These signalling pathways are involved in the pathogenesis of insulin resistance by interfering with insulin action. Many studies suggested that fluctuation of cytokines and state of signalling pathways should be considered before analysing inflammation induced obesity and insulin resistance [8].

\section{Obesity Inflammation to Insulin Resistance}

Insulin resistance is a condition in which cells fail to respond to insulin. Insulin resistance is a complex problem in which tissues, which are highly sensitive to insulin becomes less sensitive to insulin. There are three metabolic tissues, skeletal muscle, liver and white adipose tissue which becomes less sensitive under normal serum glucose conditions [9]. Insulin resistance is related to various diseases like obesity, hypoglycemia, hypertension and heart diseases. It might occur due to various disorders like insulin receptor dysfunction, inflammation and micro vascular dysfunction $[10,11]$.

Obesity is the main cause of insulin resistance. Obesity increases the lipid inflation in adipocytes due to over nutrition [9]. Excessive lipid accumulation activates c-Jun N-terminal kinase (JNK) and nuclear factor kappa B (NF- $\mathrm{kB}$ ) signalling pathways and increases the production of pro-inflammatory markers like tumour necrosis factor- $\alpha$ (TNF- $\alpha$ ) and interleukin- 6 (IL-6) [12].

\section{Cytokines Involved in Insulin Resistance}

TNF- $\alpha$

In 1990 researcher first time studied TNF- $\alpha$ and relationship of inflammation with insulin resistance [13]. TNF- $\alpha$ is one of adipose tissue derived pro-inflammatory cytokine that increases lipolysis and also causes serine/threonine phosphorylation of insulin receptor substrate-1 [12]. Among pathways activated 
by TNF- $\alpha$, activation of adenosine mono-phosphate-activated protein kinase (AMPK) increases glucose uptake in both visceral and subcutaneous adipocytes, whereas activation of JNK pathway leads to insulin resistance in visceral adipocytes [14].

\section{IL-6}

IL-6 is an inflammatory cytokine and secreted by adipose tissues and considered as the mediator that regulates insulin resistance. It increases insulin resistance by reducing expression of insulin receptor substrate- 1 and glucose transporter- 4 via Janus kinase-signal transducer and activator of transcription (JAK-STAT) signalling pathway [15]. It is also reported that IL-6 also cause insulin resistance through activation of phosphoinositide 3-kinase (PI3K) pathway by reducing microRNA expression and up-regulating GATA-2 [16,17].

\section{Leptin}

Leptin a protein which is derived from adipose tissue i.e. white adipose tissue and it intervened between appetite and energy balance and associated with progression of insulin resistance [18]. The role of leptin is not clear in insulin resistance because of Leptin resistance that is often observed in obese people, and weight loss decreases serum Leptin level. Besides this, Leptin stimulates PI3K signalling pathway that regulates glucose metabolism and pancreatic -cell function [18,19].

\section{Adiponectin}

Adiponectin is a human protein that is involved in regulation of glucose and fatty acid breakdown. It is produced by adipose tissues, primarily by white adipose tissues. It plays a dual role, as an anti-inflammatory cytokine in insulin resistance, obesity, type 2 diabetes and acts as a pro-inflammatory cytokine in osteoarthritis and type 1 diabetes [18,20]. Adiponectin receptor 2 contrarily enhances glucose consumption by regulating their gene expression via PPAR- $\alpha$ signalling pathway [21]. In short, adiponectin improves hepatic insulin resistance by making balance via reducing glycogenesis and lipogenesis and increasing glucose consumption.

\section{IL-1 $\beta$}

Interleukin- $1 \beta$ is also pro-inflammatory cytokine which secretes in the result of inflammasome activity in obesity. It also contributes to insulin resistance via impairment of insulin signalling in tissues and cells leads to impaired insulin secretion [21].

\section{Signalling Pathways Involved in Insulin Resistance}

The above described pro-inflammatory cytokines and proteins are mainly involved in insulin resistance related to inflammation induced obesity [4]. These cytokines and proteins, TNF- $\alpha$, IL-6, IL-1 $\beta$, Leptin, and Adiponectin act through JNK pathway, (JAK-STAT) signalling pathway, Inflammasome Activity, PI3K signalling pathway and PPAR- $\alpha$ signalling pathway respectively [22].
Pro-inflammatory cytokines act via transcriptional factors that regulate the expression of inflammatory genes. Among these regulatory pathways, IKK/NF- $\mathrm{kB}$ pathway is significant that regulate inflammation induced insulin resistance $[23,24]$. NF- $\mathrm{kB}$ is a transcriptional factor, a complex of proteins such as p65/RelA, RelB, c-Rel, p50/p105, and p52/p100. It is required in series of pathogenic processes including inflammation and immune responses [25].

In normal condition NF-kB is bound to IB protein in the cytoplasm. IB protein inhibits the nuclear localization of NF- $\mathrm{kB}$ in normal circumstances. During pathological problems, various stimuli trigger the IKK complex which causes phosphorylation of IB $\alpha$ on Ser32 and 36. This leads to degradation of IB $\alpha$ and triggers translocation of NF-kB to the nucleus $[25,13]$. NF-kB nucleus translocation causes the up-regulation of target genes that encode inflammatory mediators.

NF-kB mediated cytokines involve TNF- $\alpha$, IL-6, and IL$1 \beta$ that regulate the inflammatory processes which induce insulin resistance. It is noticed that lack of IKK $\beta$ in adipocytes prevents expression of TNF- $\alpha$, IL- $\beta$, and IL- 6 by free fatty acid; it inhibits the expression of anti-inflammatory cytokine leptin and adiponectin [26]. Many studies suggested that treatment that inhibits NF- $\mathrm{kB}$ always improve insulin resistance. It is also observed that NF- $\mathrm{kB}$ plays a vital role in insulin resistance related diseases that couples pro-inflammatory cytokines, like obesity and type 2 diabetes $[23,24]$. These facts bring us to the conclusion that pro-inflammatory cytokines which regulated by NF- kB signalling pathway play a central role in inflammatory process and obesity toward insulin resistance.

\section{References}

1. Segula D (2014) Complications of obesity in adults: a short review of the literature. Malawi Med J 26(1): 20-24.

2. Nuttall FQ (2015) Body mass index: obesity, BMI, and health: a critical review. Nutr Today 50(3): 117-128.

3. Lebovitz H (2001) Insulin resistance: definition and consequences. Exp Clin Endocrinol Diabetes 109(Suppl 2): S135-S148.

4. Hardy OT, Czech MP, Corvera S (2012) What causes the insulin resistance underlying obesity? Curr Opin Endocrinol Diabetes Obes 19(2): 81-87.

5. Johnson AM, Olefsky JM (2013) The origins and drivers of insulin resistance. Cell 152(4): 673-684.

6. Ye J (2009) Emerging role of adipose tissue hypoxia in obesity and insulin resistance. Int J Obes (Lond) 33(1): 54-66.

7. Trayhurn P, Wood IS (2004) Adipokines: inflammation and the pleiotropic role of white adipose tissue. Br J Nutr 92(3): 347-355.

8. Kahn SE, Hull RL, Utzschneider KM (2006) Mechanisms linking obesity to insulin resistance and type 2 diabetes. Nature 444(7121): 840-846.

9. Chawla A, Nguyen KD, Goh YS (2011) Macrophage-mediated inflammation in metabolic disease. Nat Rev Immunol 11(11): 738-749.

10. Abdul-Ghani MA, DeFronzo RA (2010) Pathogenesis of insulin resistance in skeletal muscle. BioMed Research International 2010: 476279. 
11. Chen L, Chen R, Wang H, Liang F (2015) Mechanisms linking inflammation to insulin resistance. International Journal of Endocrinology 2015(2015): 1-9.

12. Shoelson SE, Lee J, Goldfine AB (2006) Inflammation and insulin resistance. J Clin Invest 116(7): 1793-1801.

13. Lee BC, Lee J (2014) Cellular and molecular players in adipose tissue inflammation in the development of obesity-induced insulin resistance. Biochim Biophys Acta 1842(3): 446-462.

14. McArdle MA, Finucane OM, Connaughton RM, McMorrow AM, Roche HM (2013) Mechanisms of obesity-induced inflammation and insulin resistance: insights into the emerging role of nutritional strategies. Front Endocrinol (Lausanne) 4: 52.

15. Lukic L, Lalic NM, Rajkovic N, Jotic A, Lalic K, et al. (2014) Hypertension in obese type 2 diabetes patients is associated with increases in insulin resistance and IL-6 cytokine levels: potential targets for an efficient preventive intervention. Int J Environ Res Public Health 11(4): 35863598.

16. Yin J, Gao Z, He Q, Zhou D, Guo Z, et al. (2009) Role of hypoxia in obesity-induced disorders of glucose and lipid metabolism in adipose tissue. Am J Physiol Endocrinol Metab 296(2): E333-E342.

17. Yadav A, Kataria MA, Saini V (2013) Role of leptin and adiponectin in insulin resistance. Clinica Chimica Acta 417: 80-84.

18. Stofkova A (2009) Leptin and adiponectin: from energy and metabolic dysbalance to inflammation and autoimmunity. Endocr Regul 43(4): 157-168.
19. Al-Suhaimi EA, Shehzad A (2013) Leptin, resistin and visfatin: the missing link between endocrine metabolic disorders and immunity. Eur J Med Res 18: 12.

20. Carter S, Caron A, Richard D, Picard F (2013) Role of leptin resistance in the development of obesity in older patients. Clin Interv Aging 8: 829-844.

21. Chang LC, Huang KC, Wu YW, Kao HL, Chen CL (2009) The clinical implications of blood adiponectin in cardiometabolic disorders. J Formos Med Assoc 108(5): 353-366.

22. Tanti JF, Ceppo F, Jager J, Berthou F (2012) Implication of inflammatory signalling pathways in obesity-induced insulin resistance. Front Endocrinol (Lausanne) 3: 181.

23. Su D, Coudriet Gm, Hyun Kim D, Lu Y, Perdomo G, et al. (2009) Foxo1 links insulin resistance to proinflammatory cytokine IL-1 production in macrophages. Diabetes 58(11): 2624-2633.

24. Yekollu SK, Thomas R, Sullivan OB (2011) Targeting curcusomes to inflammatory dendritic cells inhibits NF-B and improves insulin resistance in obese mice. Diabetes 60(11): 2928-2938.

25. Rahman MM, McFadden G (2011) Modulation of NF-B signalling by microbial pathogens. Nat Rev Microbiol 9(4): 291-306.

26. Jiao P, Ma J, Feng B, Zhang H, Diehl JA, et al. (2011) FFA-induced adipocyte inflammation and insulin resistance: involvement of ER stress and IKK pathways. Obesity (Silver Spring) 19(3): 483-491. 